\title{
To be, or not to be finite? The Higgs potential in Gauge-Higgs Unification
}

\author{
Junji Hisano, ${ }^{a, b, c}$ Yutaro Shoji $^{a}$ and Atsuyuki Yamada ${ }^{b}$ \\ ${ }^{a}$ Kobayashi-Maskawa Institute for the Origin of Particles and the Universe, Nagoya University, \\ Furo-cho Chikusa-ku, Nagoya, 464-8602 Japan \\ ${ }^{b}$ Department of Physics, Nagoya University, \\ Furo-cho Chikusa-ku, Nagoya, 464-8602 Japan \\ ${ }^{c}$ Kavli IPMU (WPI), UTIAS, University of Tokyo, \\ Kashiwa, Chiba, 277-8584 Japan \\ E-mail: hisano@eken.phys.nagoya-u.ac.jp, yshoji@kmi.nagoya-u.ac.jp, \\ atsuyuki@eken.phys.nagoya-u.ac.jp
}

ABSTRACT: In this paper, we investigate the finiteness of the Higgs effective potential in an $\mathrm{SU}(\mathcal{N})$ Gauge-Higgs Unification (GHU) model defined on $\mathbf{M}^{4} \times S^{1}$. We obtain the Higgs effective potential at the two-loop level and find that it is finite. We also discuss that the Higgs effective potential is generically divergent for three- or higher-loop levels. As an example, we consider an $\mathrm{SU}(\mathcal{N})$ gauge theory on $\mathbf{M}^{5} \times S^{1}$, where the one-loop corrections to the four-Fermi operators are divergent. We find that the Higgs effective potential depends on their counter terms at the three-loop level.

Keywords: Phenomenology of Field Theories in Higher Dimensions

ARXIV EPRINT: 1908.09158 


\section{Contents}

1 Introduction 1

2 Dynamical symmetry breaking by Hosotani mechanism 3

3 Compactification by superposition 4

4 Higgs effective potential up to two-loop level $\quad 6$

4.1 One-loop effective potential 6

$\begin{array}{lll}4.2 & \text { Two-loop effective potential } & 7\end{array}$

$\begin{array}{llr}5 & \text { Divergences at higher-loop level } & 9\end{array}$

$\begin{array}{lll}6 & \text { Summary } & 10\end{array}$

$\begin{array}{ll}\text { A Proof of identities } & 11\end{array}$

A.1 Proof of eq. (3.5) 11

A.2 Proof of eq. (4.6) 12

A.3 Symmetries of $G_{\text {adj }}$ and $G_{\ell}$

$\begin{array}{ll}\text { B Momentum integrals } & \mathbf{1 3}\end{array}$

$\begin{array}{lll}\text { B.1 Momentum integrals with a spacial shift operator } & 13\end{array}$

$\begin{array}{ll}\text { B.2 Proof of } F(0)=0 & 14\end{array}$

$\begin{array}{ll}\text { C Example of the two-loop calculation } & 16\end{array}$

\section{Introduction}

The Higgs mechanism is one of the essential ingredients in the standard model (SM) of particle physics. It generates masses for the gauge bosons and the fermions, which were forbidden by the gauge symmetries of the standard model. Consequently, all the masses are described by the Higgs vacuum expectation value (VEV) and the couplings, which is now in good agreement with the Higgs coupling measurements at Large Hadron Collider [1, 2].

In spite of the importance of the mechanism, the nature of the Higgs boson has not been understood well. It has been discussed for a long time that a scalar field is very sensitive to a UV cutoff scale, such as the Planck scale or the grand unification scale, and it is not natural that the Higgs VEV lies around the electroweak (EW) scale. If the Higgs boson is really a fundamental scalar field, one needs to protect the Higgs mass term from dangerous quantum corrections, which is greatly achieved by supersymmetry [3-7]. Alternatively, one can assume that the Higgs boson originates from fields with non-zero 
spins. One such example is composite Higgs models [8-14], where the Higgs boson appears as a pseudo Nambu-Goldstone boson in association with the condensation of fermions. Another example, which is relevant to this paper, is the gauge-Higgs unification (GHU) [1520], where the four-dimensional gauge fields and the Higgs field are unified into gauge fields in higher-dimensional spacetime.

In the GHU, we consider gauge theories defined on non-simply connected spacetime and identify the Yang-Mills Aharonov-Bohm (AB) phases as Higgs bosons. Their tree level potential is protected because the Lagrangian has to be invariant under gauge transformations. Since the transformation variables need to be single-valued, not all of the gauge transformations are consistent with the compactification of the extra-dimensions. Thus, quantum corrections, which are sensitive to the global structure of the spacetime, generate a Higgs potential that is scaled by the compactification scale. It stabilizes the Higgs boson and breaks the gauge symmetry dynamically. This is called the Hosotani mechanism $[18,20]$.

Although gauge theories are generically non-renormalizable in more than four-dimensional spacetime, the Higgs potential might not depend on UV-theory and might be completely determined within the framework of the GHU, as conjectured in [21-23]. In fact, it has been explicitly shown that the Higgs potential is finite at the one-loop level in generic GHU models [18, 20, 24, 25] and at the two-loop level in an Abelian GHU model [26, 27]. However, it has not been clear whether the Higgs potential is finite at all orders.

To make things clear, let us state our criteria of finiteness. We allow only the gauge interactions as irrelevant operators in the tree level Lagrangian since they become relevant at the low energy four-dimensional effective field theory. The counter terms for any other divergent operators are assumed to be determined by UV-theory. From this standpoint, we claim the Higgs potential is finite if all the divergences are subtracted by the counter terms for the operators in the tree level Lagrangian. In other words, the Higgs potential is claimed to be divergent if it depends on any of the counter terms determined by UV-theory.

In this paper, we investigate the finiteness of the Higgs potential using an $\operatorname{SU}(\mathcal{N})$ gauge theory defined on $\mathbf{M}^{4} \times S^{1}$. Here, $\mathbf{M}^{4}$ represents the four-dimensional Minkowski spacetime and $S^{1}$ represents a compactified extra-dimension. Although it is the simplest manifold to realize the GHU, it is straightforward to extend our discussion to other cases.

To overcome technical difficulties that appear in perturbative calculation, we discuss a method, compactification by superposition, which greatly simplifies the calculation of the Higgs potential in a non-Abelian GHU model. A similar method has been used in the literature [28-31] for Abelian cases. In this method, momentum sums and integrals in $\mathbf{M}^{4} \times S^{1}$ are expressed as superposition of momentum integrals in $\mathbf{M}^{5}$, i.e. five-dimensional Minkowski spacetime. Thus, all the AB phases can be "gauged away" from each integral. All the information about the $\mathrm{AB}$ phases is then recovered when we superpose the results after the integration. Another virtue of this method is that the periodicity of the Higgs potential is manifest during the calculation, which would become obscure if we adopted a straightforward calculation with the Kaluza-Klein (KK) decomposition.

Using the method, we obtain the Higgs potential at the one-loop level and that at the two-loop level, which turn out to be finite. We confirm that the one-loop results agree 
with the previous works $[18,20,24]$ and the two-loop results are consistent with those for an Abelian model $[26,27]$. The two-loop finiteness in a non-Abelian model is highly non-trivial and is one of the new results in this work.

To investigate the finiteness at higher-loop levels, we increase the spacetime dimension and consider $\mathbf{M}^{5} \times S^{1}$, which allows divergences to appear in an earlier stage of loop expansions. We find that the four-Fermi operators are divergent at the one-loop level and their counter terms contribute to the Higgs potential at the three-loop level. Thus, the Higgs potential inevitably depends on UV-theory, which falsifies the conjecture for this model.

This paper is organized as follows. In section 2, we briefly review our setup and the Hosotani mechanism. In section 3, we explain our method to calculate the Higgs potential. The one-loop and the two-loop calculations of the Higgs potential are presented in section 4 . Then, we discuss the finiteness of the Higgs potential at higher-loop orders in section 5 . Finally, we summarize in section 6 .

\section{Dynamical symmetry breaking by Hosotani mechanism}

In this section, we review the Hosotani mechanism in an $\mathrm{SU}(\mathcal{N})$ gauge theory defined on $\mathbf{M}^{4} \times S^{1}$. Here, $\mathbf{M}^{4}$ is the four-dimensional Minkowski spacetime, whose coordinates are denoted by $x^{\mu}$ with $\mu \in\{0,1,2,3\}$. The fifth dimension is compactified on $S^{1}$ with radius $R$, whose coordinate is denoted by $y \in[0,2 \pi R)$. The gauge sector is described by a gauge coupling constant, $g$, gauge bosons, $A_{M}^{a}$, and its field strength, $F_{M N}^{a}$, where the capital indices, $M$ and $N$, run over $\{0,1,2,3,5\}$ and $a$ is the group index. We also introduce massless Dirac fermions, $\psi_{\ell}$, in arbitrary representations of $\mathrm{SU}(\mathcal{N})$. In the Hosotani mechanism, $A_{5}^{a}$ plays the role of the Higgs boson in the SM and its VEV is denoted as

$$
\left\langle A_{5}^{a}\right\rangle=\frac{\theta^{a}}{2 \pi R g}
$$

where $\theta^{a}$ 's are constants.

In this paper, we use the background field methods [32] in order to evaluate the effective potential of $\theta^{a}$ 's. For this purpose, we separate $A_{5}^{a}$ into the quantum and background fields as

$$
A_{5}^{a} \rightarrow A_{5}^{a}+\frac{\theta^{a}}{2 \pi R g} .
$$

The Lagrangian we consider is given by

$$
\mathcal{L}=-\frac{1}{4} F_{M N}^{a} F^{a M N}+\sum_{\ell} \bar{\psi}_{\ell} i \gamma^{M} D_{M} \psi_{\ell}+\mathcal{L}_{\mathrm{GF}}+\mathcal{L}_{\text {ghost }}
$$

where the gauge fixing terms are given by

$$
\mathcal{L}_{\mathrm{GF}}=-\frac{1}{2} \mathcal{F}^{a} \mathcal{F}^{a}
$$

with

$$
\mathcal{F}^{a}=\partial^{M} A_{M}^{a}+\frac{f^{a b c}}{2 \pi R} A_{5}^{b} \theta^{c}
$$


Here, $f^{a b c}$ is the structure constant of $\mathrm{SU}(\mathcal{N})$. The corresponding Faddeev-Popov (FP) ghost terms are given by

$$
\mathcal{L}_{\text {ghost }}=-\bar{c}^{a}\left[\partial^{M} D_{M}^{a b}-\frac{f^{a c e} f^{b e d}}{2 \pi R} \theta^{c}\left(\frac{\theta^{d}}{2 \pi R}+g A_{5}^{d}\right)\right] c^{b} .
$$

Here, the covariant derivative for an adjoint representation is given by

$$
D_{M} c \equiv\left(\partial_{M}-i g A_{M}^{a} T^{a}-i \frac{\theta^{a} T^{a}}{2 \pi R} \delta_{M}^{5}\right) c
$$

where $\left[T^{a}\right]_{b c}=-i f^{a b c}$, and that for a fermion is given by

$$
D_{M} \psi_{\ell} \equiv\left(\partial_{M}-i g A_{M}^{a} \tau_{\ell}^{a}-i \frac{\theta^{a} \tau_{\ell}^{a}}{2 \pi R} \delta_{M}^{5}\right) \psi_{\ell},
$$

where $\tau_{\ell}^{a}$ depends on the representation of $\psi_{\ell}$.

Throughout this paper, we adopt the following boundary conditions for simplicity;

$$
\begin{aligned}
A_{M}^{a}\left(x^{\mu}, y+2 \pi R\right) & =A_{M}^{a}\left(x^{\mu}, y\right), \\
\psi_{\ell}\left(x^{\mu}, y+2 \pi R\right) & =e^{i \beta_{\ell}} \psi_{\ell}\left(x^{\mu}, y\right),
\end{aligned}
$$

where $\beta_{\ell}$ 's are arbitrary phase factors.

Let us briefly explain the Hosotani mechanism using this setup. Without the boundary conditions, we could gauge away $\theta^{a}$ 's by

$$
\begin{aligned}
& A_{5}\left(x^{\mu}, y\right) \rightarrow e^{-i \frac{\theta^{a} T^{a}}{2 \pi R} y} A_{5}\left(x^{\mu}, y\right) e^{i \frac{\theta^{a} T^{a}}{2 \pi R} y}-\frac{\theta^{a} T^{a}}{2 \pi R g}, \\
& \psi_{\ell}\left(x^{\mu}, y\right) \rightarrow e^{-i \frac{\theta^{a} \tau_{\ell}^{a}}{2 \pi R} y} \psi_{\ell}\left(x^{\mu}, y\right),
\end{aligned}
$$

where $A_{M}=A_{M}^{a} T^{a}$. With the boundary conditions, however, we can gauge away $\theta^{a}$ 's only when

$$
e^{i \theta^{a} T^{a}}=\mathbb{I},
$$

where $\mathbb{I}$ is the identity matrix. Due to this constraint, $\theta^{a}$ 's become physical degrees of freedom living in a compact space labeled by $e^{i \theta^{a} T^{a}}$. Since the tree level Lagrangian is still invariant under the transformation described by eqs. (2.11) and (2.12), $\theta^{a}$ 's do not have a potential at the tree level. As we will see later, they obtain an effective potential at the one-loop level and are stabilized. If some of $\theta^{a}$ 's are non-zero at the minimum of the effective potential, they dynamically break the gauge symmetry and generate gauge boson masses.

\section{Compactification by superposition}

In the usual computation of quantum corrections in a theory with compactified extradimensions, we use the KK decomposition and evaluate four-dimensional loop integrals for 
each KK mode. For example, in an Abelian case, a typical integral at the one-loop level is given by

$$
I \equiv \frac{1}{2 \pi R} \sum_{n=-\infty}^{\infty} \int \frac{d^{4} k}{(2 \pi)^{4}}\left[k^{\mu} k_{\mu}-\left(\frac{n}{R}+\frac{\theta}{2 \pi R}\right)^{2}\right]^{-s},
$$

where $s$ is a positive constant and $n / R$ is the momentum along $S^{1}$, which labels the KK modes.

Although the KK decomposition is useful in many cases, it is not in the calculation of the effective potential of $\theta$, i.e. the Higgs boson in the GHU. Since the Higgs boson is intrinsically the $\mathrm{AB}$ phase, its effects can only be seen by particles that go around $S^{1}$ and interfere with themselves. In the KK decomposition, however, it is difficult to define the number of times the particles go around $S^{1}$ since the KK modes are momentum eigenstates.

In this paper, we discuss another way to decompose quantum fluctuations, which has been used in [28-31] for Abelian cases. The new decomposition is related to the KK decomposition by the Poisson resummation formula, ${ }^{1}$ which is given by

$$
\sum_{n=-\infty}^{\infty} 2 \pi \delta\left(k_{5}-\frac{n}{R}\right)=2 \pi R \sum_{m=-\infty}^{\infty} e^{-i 2 \pi R m k_{5}}
$$

Using this identity, eq. (3.1) becomes

$$
I=\sum_{m=-\infty}^{\infty} \int \frac{d^{5} k}{(2 \pi)^{5}} e^{-i 2 \pi R m k_{5}}\left[k^{\mu} k_{\mu}-\left(k_{5}+\frac{\theta}{2 \pi R}\right)^{2}\right]^{-s} .
$$

It implies that a loop integral in $\mathbf{M}^{4} \times S^{1}$ can be reproduced by superposition of loop integrals in $\mathbf{M}^{5}$. Since the phase factor is the shift operator of $\left(x^{\mu}, y\right) \rightarrow\left(x^{\mu}, y-2 \pi R m\right)$, we call $m$ the winding number.

Since the AB phase can be "gauged away" in $\mathbf{M}^{5}$, we can further simplify the integral as

$$
I=\sum_{m=-\infty}^{\infty} e^{i \theta m} \int \frac{d^{5} k}{(2 \pi)^{5}} e^{-i 2 \pi R m k_{5}}\left[k^{M} k_{M}\right]^{-s},
$$

by shifting $k_{5}$. In this expression, all the $\theta$-dependence appears as phase factors in association with the superposition and we can execute the loop integrals independently of $\theta$. Furthermore, the periodicity of $\theta$ is manifest.

The above decomposition is very powerful especially in a non-Abelian case, where we have the following identity;

$$
\frac{1}{2 \pi R} \sum_{n=-\infty}^{\infty} S\left(\frac{n}{R}+\frac{\Theta}{2 \pi R}\right)=\sum_{m=-\infty}^{\infty} e^{i \Theta m} \int_{-\infty}^{\infty} \frac{d k_{5}}{2 \pi} e^{-i 2 \pi R k_{5} m} S\left(k_{5}\right),
$$

where $\Theta$ is a Hermitian matrix and $S(\ldots)$ is an analytic function or its extension to a matrix function (we call it as an "analytic function" in short). We provide its proof in

\footnotetext{
${ }^{1}$ It is essentially the same transformation as is used in the previous calculations [18, 20, 24], where it is applied after the four-dimensional integration. We apply it before the four-dimensional integration and promote it to a five-dimensional one.
} 
appendix A.1. It removes all the matrix-valued objects from momentum integrals and simplifies the calculation enormously.

In this paper, we do not try to construct the Feynman rules that generate the final expressions directly. Instead, we first use the KK decomposition and then convert the expressions by eq. (3.5).

\section{Higgs effective potential up to two-loop level}

In this section, we calculate the one-loop and the two-loop effective Higgs potentials explicitly and show that they are finite.

\subsection{One-loop effective potential}

At the one-loop level, the quantum corrections to the Higgs effective potential from the gauge bosons, the FP ghosts and the fermions can be calculated from

$$
\begin{aligned}
& V_{A, \mathrm{eff}}^{1 \mathrm{~L}}(\theta)=i\left\{=-\frac{5 i}{2} \frac{1}{2 \pi R} \sum_{n} \int \frac{d^{4} k}{(2 \pi)^{4}} \operatorname{tr} \ln \left[k^{2}-\left(\frac{n}{R}+\frac{\theta^{a} T^{a}}{2 \pi R}\right)^{2}\right],\right. \\
& V_{c, \mathrm{eff}}^{1 \mathrm{~L}}(\theta)=i \vdots=i \frac{1}{2 \pi R} \sum_{n} \int \frac{d^{4} k}{(2 \pi)^{4}} \operatorname{tr} \ln \left[k^{2}-\left(\frac{n}{R}+\frac{\theta^{a} T^{a}}{2 \pi R}\right)^{2}\right], \\
& V_{F, \mathrm{eff}}^{1 \mathrm{~L}}(\theta)=i \bigcirc=\sum_{\ell} 2 i \frac{1}{2 \pi R} \sum_{n} \int \frac{d^{4} k}{(2 \pi)^{4}} \operatorname{tr} \ln \left[k^{2}-\left(\frac{n}{R}+\frac{\theta^{a} \tau_{\ell}^{a}-\beta_{\ell}}{2 \pi R}\right)^{2}\right],
\end{aligned}
$$

respectively. Here after, all the sums except for those of the flavor index, $\ell$, are taken from $-\infty$ to $\infty$ if not explicitly specified. We convert them with eq. (3.5) as

$$
\begin{aligned}
& \frac{1}{2 \pi R} \sum_{n} \int \frac{d^{4} k}{(2 \pi)^{4}} \operatorname{tr} \ln \left[k^{2}-\left(\frac{n}{R}+\frac{\Theta}{2 \pi R}\right)^{2}\right] \\
& =-\lim _{s \rightarrow 0} \frac{d}{d s} \frac{1}{2 \pi R} \sum_{n} \int \frac{d^{4} k}{(2 \pi)^{4}} \operatorname{tr}\left[k^{2}-\left(\frac{n}{R}+\frac{\Theta}{2 \pi R}\right)^{2}\right]^{-s} \\
& =-\lim _{s \rightarrow 0} \frac{d}{d s} \sum_{m} \operatorname{tr} e^{i \Theta m} \int \frac{d^{5} k}{(2 \pi)^{5}} e^{-i 2 \pi R m k_{5}}\left(k^{M} k_{M}\right)^{-s},
\end{aligned}
$$

where $\Theta=\theta^{a} T^{a}$ or $\Theta=\theta^{a} \tau_{\ell}^{a}-\beta_{\ell}$. The loop integrals are executed in appendix B and we get

$$
\lim _{s \rightarrow 0} \frac{d}{d s} \int \frac{d^{5} k}{(2 \pi)^{5}} e^{-i 2 \pi R m k_{5}}\left(k^{M} k_{M}\right)^{-s}=\frac{3 i}{128|m|^{5} \pi^{7} R^{5}}
$$

for $m \neq 0$. Thus, we get

$$
V_{\mathrm{eff}}^{1 \mathrm{~L}}(\theta)=-\frac{9}{256 \pi^{7} R^{5}} \sum_{m \neq 0} \frac{1}{|m|^{5}} \operatorname{tr} e^{i \theta^{a} T^{a} m}+\frac{3}{64 \pi^{7} R^{5}} \sum_{\ell} \sum_{m \neq 0} \frac{1}{|m|^{5}} \operatorname{tr} e^{i\left(\theta^{a} \tau_{\ell}^{a}-\beta_{\ell}\right) m}+C,
$$

where $C$ represents the $\theta$-independent divergent terms, i.e. the contributions from $m=0$. The $\theta$-dependent part is finite and consistent with the previous works [18, 20, 24]. 


\subsection{Two-loop effective potential}

At the two-loop level, we need to work a little more because we cannot directly use eq. (3.5) to convert expressions. In the calculation, we often face the following expression;

$$
\frac{1}{(k+p)^{\mu}(k+p)_{\mu}-\left(\frac{n+n^{\prime}}{R}+\frac{\theta^{a} \tau^{a}-\beta}{2 \pi R}\right)^{2}} \tau^{b} \frac{1}{k^{\mu} k_{\mu}-\left(\frac{n}{R}+\frac{\theta^{a} \tau^{a}-\beta}{2 \pi R}\right)^{2}},
$$

where $\tau^{a}$ 's are generators of $\mathrm{SU}(\mathcal{N})$. It is not an analytic function of $\left(\frac{n}{R}+\frac{\tau^{a} \theta^{a}-\beta}{2 \pi R}\right)$ since we have $\tau^{b}$ in the middle. To remove $\tau^{b}$, we use

$$
S\left(\theta^{a} \tau^{a}\right) \tau^{b}=\tau^{c}\left[S\left(\theta^{a} \tau^{a}+\theta^{a} T^{a}\right)\right]_{c b},
$$

where $S(\ldots)$ is an arbitrary analytic function. Here, the indices in the subscript are those for $T^{a}$, not for $\tau^{a}$. Its proof is given in appendix A.2. Then, the expression becomes

$$
\tau^{c}\left[\frac{1}{(k+p)^{\mu}(k+p)_{\mu}-\left(\frac{n+n^{\prime}}{R}+\frac{\theta^{a} \tau^{a}-\beta+\theta^{a} T^{a}}{2 \pi R}\right)^{2}} \frac{1}{k^{\mu} k_{\mu}-\left(\frac{n}{R}+\frac{\theta^{a} \tau^{a}-\beta}{2 \pi R}\right)^{2}}\right]_{c b} .
$$

Now, the inside of the square brackets can be seen as an analytic function of $\left(\frac{n}{R}+\frac{\tau^{a} \theta^{a}-\beta}{2 \pi R}\right)$ for each $(c, b),{ }^{2}$ and we can apply eq. (3.5). We show an example of the two-loop calculation in appendix $\mathrm{C}$.

There are four diagrams at the two-loop level. ${ }^{3}$ After applying eq. (3.5), we obtain the following expressions.

i) A fermion loop with a gauge boson ladder:

$$
\begin{gathered}
V_{F, \mathrm{eff}}^{2 L}(\theta)=i \circlearrowleft و^{2} \sum_{m_{1}, m_{2}} G_{\ell}\left(m_{1}, m_{2}\right) \int \frac{d^{5} p}{(2 \pi)^{5}} \int \frac{d^{5} k}{(2 \pi)^{5}} e^{-i 2 \pi R\left(p_{5} m_{1}+k_{5} m_{2}\right)} \\
\times \frac{(k+p)^{M} k_{M}}{p^{N} p_{N}(k+p)^{L}(k+p)_{L} k^{K} k_{K}},
\end{gathered}
$$

where

$$
G_{\ell}\left(m_{1}, m_{2}\right) \equiv\left[e^{i \theta^{c} T^{c} m_{1}}\right]_{b a} \operatorname{tr}\left[e^{i\left(\theta^{c} \tau_{\ell}^{c}-\beta_{\ell}\right) m_{2}} \tau_{\ell}^{a} \tau_{\ell}^{b}\right] .
$$

ii) A ghost loop with a gauge boson ladder:

$$
\begin{gathered}
V_{c, \text { eff }}^{2 L}(\theta)=i \stackrel{1}{\because}=-\frac{1}{2} g^{2} \sum_{m_{1}, m_{2}} G_{\text {adj }}\left(m_{1}, m_{2}\right) \int \frac{d^{5} p}{(2 \pi)^{5}} \int \frac{d^{5} k}{(2 \pi)^{5}} e^{-i 2 \pi R\left(p_{5} m_{1}+k_{5} m_{2}\right)} \\
\times \frac{(k+p)^{M} k_{M}}{p^{N} p_{N}(k+p)^{L}(k+p)_{L} k^{K} k_{K}}
\end{gathered}
$$

\footnotetext{
${ }^{2}$ It becomes more visible if we diagonalize $\theta^{a} T^{a}$.

${ }^{3}$ The one-loop counter term for a wave function does not contribute to the Higgs potential at the two-loop level since the $\theta$-dependence is completely canceled between the propagator and the counter term.
} 
where

$$
G_{\text {adj }}\left(m_{1}, m_{2}\right) \equiv\left[e^{i \theta^{c} T^{c} m_{1}}\right]_{b a} \operatorname{tr}\left[e^{i \theta^{c} T^{c} m_{2}} T^{a} T^{b}\right] .
$$

iii) A gauge boson loop with a gauge boson ladder:

$$
\begin{gathered}
V_{A 1, \mathrm{eff}}^{2 L}(\theta)=i \text { in } \sum_{m_{1}, m_{2}} G_{\mathrm{adj}}\left(m_{1}, m_{2}\right) \int \frac{d^{5} p}{(2 \pi)^{5}} \int \frac{d^{5} k}{(2 \pi)^{5}} e^{-i 2 \pi R\left(p_{5} m_{1}+k_{5} m_{2}\right)} \\
\times \frac{k^{M} k_{M}+k^{M} p_{M}+p^{M} p_{M}}{p^{N} p_{N}(k+p)^{L}(k+p)_{L} k^{K} k_{K}} .
\end{gathered}
$$

iv) Gauge boson loops connected by a four-point vertex:

$$
\begin{gathered}
V_{A 2, \mathrm{eff}}^{2 L}(\theta)=i\{\}=-5 g^{2} \sum_{m_{1}, m_{2}} G_{\text {adj }}\left(m_{1}, m_{2}\right) \int \frac{d^{5} p}{(2 \pi)^{5}} \int \frac{d^{5} k}{(2 \pi)^{5}} e^{-i 2 \pi R\left(p_{5} m_{1}+k_{5} m_{2}\right)} \\
\times \frac{1}{k^{M} k_{M} p^{N} p_{N}} .
\end{gathered}
$$

These loop integrals can be decomposed as

$$
\begin{aligned}
& \int \frac{d^{5} p}{(2 \pi)^{5}} \int \frac{d^{5} k}{(2 \pi)^{5}} e^{-i 2 \pi R\left(p_{5} m_{1}+k_{5} m_{2}\right)} \frac{a k^{M} k_{M}+2 b k^{M} p_{M}+c p^{M} p_{M}}{p^{N} p_{N}(k+p)^{L}(k+p)_{L} k^{K} k_{K}} \\
& \quad=-b F\left(m_{1}\right) F\left(m_{2}\right)-(a-b) F\left(m_{1}-m_{2}\right) F\left(m_{2}\right)-(c-b) F\left(m_{1}\right) F\left(m_{2}-m_{1}\right)
\end{aligned}
$$

where

$$
F(m) \equiv i \int \frac{d^{5} k}{(2 \pi)^{5}} \frac{e^{-i 2 \pi R k_{5} m}}{k^{K} k_{K}}= \begin{cases}\frac{1}{64 \pi^{5}|m|^{3} R^{3}}, & m \neq 0 \\ 0, & m=0 .\end{cases}
$$

These integrals are executed in appendix B, where we also show that $F(0)$ should vanish if we use a regularization that is consistent with gauge invariance.

In appendix A.3, we see that $G_{\text {adj }}\left(m_{1}, m_{2}\right)$ and $G_{\ell}\left(m_{1}, m_{2}\right)$ are symmetric under $m_{1} \leftrightarrow$ $m_{2}-m_{1}$, and that $G_{\text {adj }}\left(m_{1}, m_{2}\right)$ is also symmetric under $m_{1} \leftrightarrow-m_{2}$.

Using these, we get the two-loop effective potential as

$$
\begin{aligned}
V_{\text {eff }}^{2 L}(\theta)= & -3 g^{2} \sum_{\ell} \sum_{m_{1}, m_{2}} G_{\ell}\left(m_{1}, m_{2}\right)\left[2 F\left(m_{1}\right) F\left(m_{2}\right)-F\left(m_{1}\right) F\left(m_{2}-m_{1}\right)\right] \\
& +\frac{9}{4} g^{2} \sum_{m_{1}, m_{2}} G_{\text {adj }}\left(m_{1}, m_{2}\right) F\left(m_{1}\right) F\left(m_{2}\right) .
\end{aligned}
$$

As we can see, the result is finite. The Abelian case can be obtained by $T^{a} \rightarrow 0$ and $\tau_{\ell}^{a} \rightarrow Q_{\ell}$ with $Q_{\ell}$ being the $\mathrm{U}(1)$-charge of $\psi_{\ell}$. The result is consistent with the previous works $[26,27]$. 


\section{Divergences at higher-loop level}

In the previous section, we have seen that the Higgs effective potential is finite up to the two-loop level. At the one-loop level, the results are finite because we need a non-zero winding number to get $\theta$-dependent contributions. At the two-loop level, it is because of the gauge invariance for the gauge boson self-energy. However, there seems to be no reason that divergences should vanish at higher-loop levels. Since the theory is non-renormalizable, we need infinite number of counter terms, such as that for the four-Fermi operators. Connecting the external lines of such counter terms, one can easily get $\theta$-dependent contributions. Thus, if there is no non-trivial cancellation, the Higgs effective potential depends on such counter terms and hence on UV-theory. In this section, we show an example of such divergences.

Since gauge theory in $\mathbf{M}^{4} \times S^{1}$ lies around the boundary of renormalizable and nonrenormalizable theories, the divergences appear at rather higher-loop levels and it is a little hard to test the finiteness explicitly. Thus, we increase the spacial dimension and consider $\mathbf{M}^{5} \times S^{1}$. To improve visibility, we consider only one massless Dirac fermion and suppress the flavor index, $\ell$. The one-loop and the two-loop contributions are parallel to the previous discussion and can be shown to be finite.

In this example, we concentrate on the four-Fermi operator and show that the Higgs effective potential depends on its counter term. The one-loop corrections to the four-Fermi operator are log-divergent and the divergent part is calculated as

$$
\begin{aligned}
& \text { ? } \\
& =\frac{-i g^{4}}{768 \pi^{3}} \frac{1}{\epsilon}\left[\gamma^{L} \gamma^{N} \gamma^{M} \tau^{c} \tau^{a}\right]_{\alpha \beta}\left[\gamma_{M} \gamma_{N} \gamma_{L} \tau^{a} \tau^{c}-\gamma_{L} \gamma_{N} \gamma_{M} \tau^{c} \tau^{a}\right]_{\gamma \delta}-(\alpha \leftrightarrow \gamma),
\end{aligned}
$$

where $\alpha$ and $\gamma$ are spin indices of $\bar{\psi}$, and $\beta$ and $\delta$ are those of $\psi$. Here, (crossed) represents the same diagrams with the fermion lines being crossed. We have used the dimensional regularization and $\epsilon=3-D / 2$ with $D$ being the spacetime dimension.

To subtract the divergence, we need the following counter term;

$$
\mathcal{L}_{\mathrm{CT}}=\frac{\delta_{4 F}}{2}\left[\bar{\psi} \gamma^{M} \gamma^{N} \gamma^{L} \tau^{a} \tau^{b} \psi\right]\left[\bar{\psi}\left(\gamma_{M} \gamma_{N} \gamma_{L} \tau^{a} \tau^{b}-\gamma_{L} \gamma_{N} \gamma_{M} \tau^{b} \tau^{a}\right) \psi\right],
$$

where

$$
\delta_{4 F}=\frac{g^{4}}{768 \pi^{3}} \frac{1}{\epsilon}+\delta_{4 F}^{\mathrm{fin}} .
$$

Here, $\delta_{4 F}^{\text {fin }}$ represents finite renormalization and is determined by UV-theory. ${ }^{4}$

By connecting the fermion lines of the counter term, we get a finite contribution to the Higgs effective potential as

$$
\begin{aligned}
V_{\mathrm{CT}}(\theta)= & \sum_{m_{1} \neq 0} \sum_{m_{2} \neq 0} \frac{\delta_{4 F}^{\mathrm{fin}} \mathcal{N}}{8 \pi^{16} R^{10} m_{1}^{5} m_{2}^{5}} \\
& \times\left\{2 \operatorname{tr}\left[\tau^{a} e^{i\left(\theta^{b} \tau^{b}-\beta\right) m_{1}}\right] \operatorname{tr}\left[\tau^{a} e^{i\left(\theta^{b} \tau^{b}-\beta\right) m_{2}}\right]+\operatorname{tr}\left[\tau^{a} e^{i\left(\theta^{b} \tau^{b}-\beta\right) m_{1}} \tau^{a} e^{i\left(\theta^{b} \tau^{b}-\beta\right) m_{2}}\right]\right\} .
\end{aligned}
$$

\footnotetext{
${ }^{4}$ Since the four-Fermi operator is not forbidden by any symmetry, $\delta_{4 F}^{\text {fin }}$ is arbitrary.
} 
It is non-vanishing and has non-trivial $\theta$-dependence. For example, in the $\mathrm{SU}(2)$ gauge theory with a fermion in the fundamental representation, the above contribution can be expressed as

$$
V_{\mathrm{CT}}^{\mathcal{N}=2}(\theta)=\sum_{m_{1} \neq 0} \sum_{m_{2} \neq 0} \frac{3 \delta_{4 F}^{\mathrm{fin}} e^{-i \beta\left(m_{1}+m_{2}\right)}}{8 \pi^{16} R^{10} m_{1}^{5} m_{2}^{5}} \cos \left(\frac{m_{1}+m_{2}}{2} \sqrt{\left(\theta^{1}-2 \beta\right)^{2}+\left(\theta^{2}\right)^{2}+\left(\theta^{3}\right)^{2}}\right),
$$

which is indeed non-vanishing. Notice that there is only one Wilson line phase in the $\mathrm{SU}(2)$ case, which corresponds to $\sqrt{\cdots}$ in the above equation. Thus, the Higgs effective potential inevitably depends on UV-theory. It falsifies the conjecture of all-order finiteness in this model..$^{5}$

Notice that the above contribution cannot be canceled by any other diagrams. To see this, let us integrate out the fermions since we are not interested in external fermion lines. Since all of the fermion legs should be connected, the contribution of the counter term to any operator in the effective action starts at the three-loop level. There is only one diagram that contributes to the Higgs potential at the three-loop level, which is what we calculated above. One might afraid that the renormalization of the gauge coupling constant or the self energies may affect the result. However, since the modification of the renormalization starts at the three-loop level, their contribution to the Higgs potential appears at four- or higher-loop levels.

The above example implies that there is no special mechanism that prevents the Higgs effective potential to diverge. Since there are infinite number of counter terms, we expect that the effective potential is generically divergent at three- or higher-loop levels also in other models.

Although the Higgs effective potential seems to be divergent, it is notable that the divergence is suppressed at least at the three-loop level. Since the gauge theory is nonrenormalizable, we expect that it is UV-completed at a scale that is not so far from $1 / R$. Thus, such a higher-loop suppression can be strong enough to explain the little hierarchy between these scales.

\section{Summary}

In this paper, we have investigated the finiteness of the Higgs effective potential in a nonAbelian GHU model defined on $\mathbf{M}^{4} \times S^{1}$. Although the model is non-renormalizable, the Higgs effective potential is known to be finite at the one-loop level and it has been conjectured that it might be free from divergences at all orders in perturbative expansions. However, the calculation of the effective potential beyond the one-loop level has been a technical challenge and only the two-loop calculation in an Abelian model is available in the literature $[26,27]$.

To overcome the technical difficulties, we presented a powerful method to calculate the loop integrals in the GHU, compactification by superposition. We express a loop integral

\footnotetext{
${ }^{5}$ This result is not strong enough to rule out the all-order finiteness for an Abelian case since $V_{\mathrm{CT}}(\theta)$ vanishes identically. In addition, we do not exclude the possibility that the effective potential becomes finite at all-order after the inclusion of $\delta_{4 F}$. However, it is beyond our criteria of finiteness.
} 
and sum in $\mathbf{M}^{4} \times S^{1}$ as a superposition of loop integrals in $\mathbf{M}^{5}$, which allows us to remove all the matrix valued objects from the integrals. The Higgs dependence of the potential is then expressed as phase factors in association with the superposition, where the periodicity of the Higgs potential is manifest.

Using the method, we have determined the effective potential up to the two-loop level in the non-Abelian model, which turned out to be finite.

We have also discussed that the Higgs effective potential are generically divergent at the three- or higher-loop levels. As an example, we have considered an $\mathrm{SU}(\mathcal{N})$ gauge theory on $\mathbf{M}^{5} \times S^{1}$. We have seen that the one-loop correction to the four-Fermi operator is divergent and we need a counter term to renormalize the theory. Then, we have explicitly shown that the Higgs effective potential depends on the counter term at the three-loop level, which falsifies the conjecture of the all-order finiteness for this model. It seems that this feature is generic since there are infinite number of counter terms and one can easily generate the Higgs potential by connecting their legs.

Although the effective potential seems to be divergent, it is found to be suppressed at least at the three-loop level. Such higher-loop suppression is still useful to explain the hierarchy between the scale of the GHU and that of a UV cutoff.

\section{Acknowledgments}

This work was supported by Grant-in-Aid for Scientific research from the Ministry of Education, Science, Sports, and Culture (MEXT), Japan, No. 16H06492 [J.H. and Y.S.]. The work of J.H. is also supported by World Premier International Research Center Initiative (WPI Initiative), MEXT, Japan.

\section{A Proof of identities}

\section{A.1 Proof of eq. (3.5)}

Let $\Theta$ be a Hermitian matrix. Then, for an arbitrary analytic function, $S(\ldots)$, the following identity holds.

$$
\frac{1}{2 \pi R} \sum_{n=-\infty}^{\infty} S\left(\frac{n}{R}+\frac{\Theta}{2 \pi R}\right)=\sum_{n=-\infty}^{\infty} e^{i \Theta n} \int_{-\infty}^{\infty} \frac{d k_{5}}{2 \pi} S\left(k_{5}\right) e^{-i 2 \pi R k_{5} n} .
$$

Proof. We first diagonalize $\Theta$ as

$$
U^{-1} \Theta U=\operatorname{diag}\left(v_{1}, v_{2}, \cdots\right),
$$

with unitary matrix $U$. Since $S(\ldots)$ is an analytic function, we have

$$
\left[S\left(\frac{n}{R}+\frac{\Theta}{2 \pi R}\right)\right]_{a b}=U_{a c} S\left(\frac{n}{R}+\frac{v_{c}}{2 \pi R}\right) U_{c b}^{-1} .
$$

Inserting an identity, we get

$$
\left[S\left(\frac{n}{R}+\frac{\Theta}{2 \pi R}\right)\right]_{a b}=\int_{-\infty}^{\infty} \frac{d k_{5}}{2 \pi} S\left(k_{5}\right) U_{a c} 2 \pi \delta\left(k_{5}-\frac{n}{R}-\frac{v_{c}}{2 \pi R}\right) U_{c b}^{-1} .
$$


Since we have

$$
\sum_{n=-\infty}^{\infty} 2 \pi \delta\left(p-\frac{n}{R}\right)=2 \pi R \sum_{n=-\infty}^{\infty} e^{-i 2 \pi R p n}
$$

we get

$$
\sum_{n=-\infty}^{\infty}\left[S\left(\frac{n}{R}+\frac{\Theta}{2 \pi R}\right)\right]_{a b}=2 \pi R \int_{-\infty}^{\infty} \frac{d k_{5}}{2 \pi} S\left(k_{5}\right) \sum_{n=-\infty}^{\infty} U_{a c} e^{-i 2 \pi R\left(k_{5}-\frac{v_{c}}{2 \pi R}\right) n} U_{c b}^{-1}
$$

Using

$$
e^{-i 2 \pi R\left(k_{5}-\frac{v_{c}}{2 \pi R}\right) n} \delta_{c d}=\left[e^{-i 2 \pi R\left(k_{5}-\frac{\operatorname{diag}\left(v_{1}, v_{2}, \cdots\right)}{2 \pi R}\right) n}\right]_{c d}
$$

we get

$$
\sum_{n=-\infty}^{\infty} S\left(\frac{n}{R}+\frac{\Theta}{2 \pi R}\right)=2 \pi R \int_{-\infty}^{\infty} \frac{d k_{5}}{2 \pi} \sum_{n=-\infty}^{\infty} e^{-i 2 \pi R\left(k_{5}-\frac{\Theta}{2 \pi R}\right) n} S\left(k_{5}\right) .
$$

\section{A.2 Proof of eq. (4.6)}

Let $\tau^{a}$ 's be an arbitrary representation of $\mathrm{SU}(\mathcal{N}), \lambda^{a}$ 's be constants and $S(\ldots)$ be an arbitrary analytic function. Then, the following identity holds;

$$
S\left(\lambda^{a} \tau^{a}\right) \tau^{b}=\tau^{c}\left[S\left(\lambda^{a} \tau^{a}+\lambda^{a} T^{a}\right)\right]_{c b},
$$

where the indices in the subscript are those for $T^{a}$, not for $\tau^{a}$.

Proof. Since $S(\ldots)$ can be expanded locally, it is enough to prove for the case where $S(\ldots)$ is a monomial function. Since we have

$$
\left[\lambda^{a} \tau^{a}, \tau^{b}\right]=\tau^{c}\left(\lambda^{a} T_{c b}^{a}\right)
$$

we have

$$
\begin{aligned}
\left(\lambda^{a} \tau^{a}\right)^{n} \tau^{b} & =\left(\lambda^{a} \tau^{a}\right)^{n-1} \tau^{c}\left[\delta^{c b} \lambda^{a} \tau^{a}+\lambda^{a} T_{c b}^{a}\right] \\
& =\cdots=\tau^{c}\left[\lambda^{a} \tau^{a}+\lambda^{a} T^{a}\right]_{c b}^{n}
\end{aligned}
$$

Since the above holds for each term of the Taylor series, the same holds for $S(\ldots)$.

\section{A.3 Symmetries of $G_{\text {adj }}$ and $G_{\ell}$}

Let $\tau^{a}$ 's be an arbitrary representation of $\mathrm{SU}(\mathcal{N})$ and $\lambda^{a}$ 's and $\bar{\lambda}^{a}$ 's be constants. Then, the following identities hold;

$$
\begin{aligned}
{\left[e^{i \lambda^{c} T^{c}}\right]_{b a} \operatorname{tr}\left[e^{i \bar{\lambda}^{c} \tau^{c}} \tau^{a} \tau^{b}\right] } & =\left[e^{i\left(\bar{\lambda}^{c}-\lambda^{c}\right) T^{c}}\right]_{b a} \operatorname{tr}\left[e^{\left.i \bar{\lambda}^{c} \tau^{c} \tau^{a} \tau^{b}\right]}\right. \\
{\left[e^{i \lambda^{c} T^{c}}\right]_{b a} \operatorname{tr}\left[e^{i \bar{\lambda}^{c} T^{c}} T^{a} T^{b}\right] } & =\left[e^{-i \bar{\lambda}^{c} T^{c}}\right]_{b a} \operatorname{tr}\left[e^{-i \lambda^{c} T^{c}} T^{a} T^{b}\right]
\end{aligned}
$$


Proof. The first identity can be shown by using the identity of appendix A.2. We have

$$
\begin{aligned}
{\left[e^{i \lambda^{c} T^{c}}\right]_{b a} \operatorname{tr}\left[e^{i \bar{\lambda}^{c} \tau^{c}} \tau^{a} \tau^{b}\right] } & =\left[e^{i \lambda^{c} T^{c}}\right]_{b a}\left[e^{i \bar{\lambda}^{c} T^{c}}\right]_{d a} \operatorname{tr}\left[\tau^{d} e^{i \bar{\lambda}^{c} \tau^{c}} \tau^{b}\right] \\
& =\left[e^{i \lambda^{c} T^{c}}\right]_{b a}\left[e^{-i \bar{\lambda}^{c} T^{c}}\right]_{a d} \operatorname{tr}\left[\tau^{d} e^{i \bar{\lambda}^{c} \tau^{c}} \tau^{b}\right] \\
& =\left[e^{i\left(\bar{\lambda}^{c}-\lambda^{c}\right) T^{c}}\right]_{d b} \operatorname{tr}\left[e^{i \bar{\lambda}^{c} \tau^{c}} \tau^{b} \tau^{d}\right]
\end{aligned}
$$

The second identity can be shown as

$$
\begin{aligned}
{\left[e^{i \lambda^{c} T^{c}}\right]_{b a} \operatorname{tr}\left[e^{i \overline{\bar{\lambda}^{c}} T^{c}} T^{a} T^{b}\right] } & =\left[e^{i \lambda^{c} T^{c}}\right]_{b a}\left[e^{i \bar{\lambda}^{c} T^{c}}\right]_{c d} T_{d e}^{a} T_{e c}^{b} \\
& =\left[e^{-i \lambda^{c} T^{c}}\right]_{a b}\left[e^{-i \bar{\lambda}^{c} T^{c}}\right]_{d c} T_{b e}^{c} T_{e a}^{d} \\
& =\left[e^{-i \overline{\lambda^{c}} T^{c}}\right]_{d c} \operatorname{tr}\left[e^{-i \lambda^{c} T^{c}} T^{c} T^{d}\right]
\end{aligned}
$$

\section{B Momentum integrals}

\section{B.1 Momentum integrals with a spacial shift operator}

In this appendix, we calculate

$$
\mathcal{I}=\int \frac{d^{D} k}{(2 \pi)^{D}}\left(-k^{M} k_{M}+2 p^{M} k_{M}+m^{2}-i \epsilon\right)^{-s} e^{-i 2 k^{M} x_{M}} .
$$

From the definition of the gamma function, we have

$$
W^{-s}=\frac{i^{s}}{\Gamma(s)} \int_{0}^{\infty} d t e^{-i W t} t^{s-1},
$$

for $\operatorname{Im}(W)<0$ and $\operatorname{Re}(s)>0$. Using this, we have

$$
\begin{aligned}
\mathcal{I} & =\frac{i^{s}}{\Gamma(s)} \int_{0}^{\infty} d t t^{s-1} \int \frac{d^{D} k}{(2 \pi)^{D}} e^{i\left(k^{M} k_{M}-2 p^{M} k_{M}-m^{2}+i \epsilon\right) t-i 2 k^{M} x_{M}} \\
& =\frac{i^{s}}{\Gamma(s)} e^{-i 2 p^{M} x_{M}} \int_{0}^{\infty} d t t^{s-1} e^{-i \frac{x^{M} x_{M}}{t}-i\left(p^{M} p_{M}+m^{2}\right) t-\epsilon t} \int \frac{d^{D} k}{(2 \pi)^{D}} e^{i k^{M} k_{M} t} \\
& =\frac{i^{s-D / 2+1}}{\Gamma(s)(4 \pi)^{D / 2}} e^{-i 2 p^{M} x_{M}} \int_{0}^{\infty} d t t^{s-D / 2-1} e^{-i \frac{x^{M} x_{M}}{t}-i\left(p^{M} p_{M}+m^{2}\right) t-\epsilon t}
\end{aligned}
$$

The integral can be evaluated as

$$
\lim _{\delta \rightarrow+0} \int_{0}^{\infty} d t t^{r-1} e^{-i B t+i \frac{C}{t}-\epsilon t-\frac{\delta}{t}}=2(-i)^{r / 2} \frac{C^{r / 2}}{[i(B-i \epsilon)]^{r / 2}} K_{r}\left(-2 i^{3 / 2} \sqrt{i C(B-i \epsilon)}\right),
$$

for $\epsilon>0, C>0$, where $K_{n}(z)$ is the modified Bessel function of the second kind. Here, we introduced a regulator $\delta>0$. 
When $0<\operatorname{Re}(s)$ and $p^{M} p_{M}+m^{2} \neq 0$, the integral is convergent and is evaluated as

$$
\begin{aligned}
\mathcal{I}= & \frac{2 i^{s / 2-D / 4+1}}{(4 \pi)^{D / 2} \Gamma(s)} \frac{e^{-i 2 p^{M} x_{M}}\left(-x^{M} x_{M}\right)^{s / 2-D / 4}}{\left[i\left(p^{M} p_{M}+m^{2}-i \epsilon\right)\right]^{s / 2-D / 4}} \\
& \times K_{s-D / 2}\left(-2 i^{3 / 2} \sqrt{i\left(p^{M} p_{M}+m^{2}-i \epsilon\right)\left(-x^{M} x_{M}\right)}\right) .
\end{aligned}
$$

Notice that

$$
K_{n+1 / 2}(x)=K_{-n-1 / 2}(x)=\left(\frac{\pi}{2 x}\right)^{1 / 2} e^{-x} \sum_{r=0}^{n} \frac{(n+r) !}{r !(n-r) !}(2 x)^{-r},
$$

with $n$ being a positive integer.

When $p^{M} p_{M}+m^{2}=0$, we need to take $B \rightarrow 0$ before $\epsilon \rightarrow 0$, which gives

$$
\lim _{B \rightarrow 0} \lim _{\delta \rightarrow+0} \int_{0}^{\infty} d t t^{r-1} e^{-i B t+i \frac{C}{t}-\epsilon t-\frac{\delta}{t}}=C^{r}(-i)^{r} \Gamma(-r)+\mathcal{O}(\epsilon)
$$

When $0<\operatorname{Re}(s)<\frac{D}{2}$ and $p^{M} p_{M}+m^{2}=0$, it becomes

$$
\mathcal{I}=\frac{i}{(4 \pi)^{D / 2}} \frac{\Gamma\left(\frac{D}{2}-s\right)}{\Gamma(s)} e^{-i 2 p^{M} x_{M}}\left(-x^{M} x_{M}\right)^{s-D / 2} .
$$

\section{B.2 Proof of $F(0)=0$}

We assume a regularization that has the following features.

- All the integrals become finite.

- Invariance under the shifts of loop momenta.

- Independence of the signs of loop momenta.

- Gauge invariance, $p_{M} \Pi^{M N}(p)=0$.

Then, the following identity holds;

$$
F(0) \equiv i \int \frac{d^{5} k}{(2 \pi)^{5}} \frac{1}{k^{K} k_{K}}=0 .
$$

Proof. Let us define

$$
\begin{aligned}
\Lambda^{3} & \equiv-i F(0)=\int \frac{d^{5} k}{(2 \pi)^{5}} \frac{1}{k^{M} k_{M}}, \\
\Xi(p) & \equiv \int \frac{d^{5} k}{(2 \pi)^{5}} \frac{1}{(k+p / 2)^{M}(k+p / 2)_{M}(k-p / 2)^{N}(k-p / 2)_{N}} .
\end{aligned}
$$

Then, we have the following relations;

$$
\begin{aligned}
& \int \frac{d^{5} p}{(2 \pi)^{5}} \Xi(p)=\left(\Lambda^{3}\right)^{2}, \\
& \int \frac{d^{5} k}{(2 \pi)^{5}} \frac{k^{M} k^{N}}{(k+p / 2)^{L}(k+p / 2)_{L}(k-p / 2)^{K}(k-p / 2)_{K}} \\
& \quad=\left(\frac{1+x}{5} \eta^{M N}-x \frac{p^{M} p^{N}}{p^{L} p_{L}}\right)\left[\Lambda^{3}-\frac{p^{L} p_{L}}{4} \Xi(p)\right],
\end{aligned}
$$

where $x$ is a constant, which will be determined later. 
At the one-loop level, the divergent corrections to the gauge boson self-energy are given by

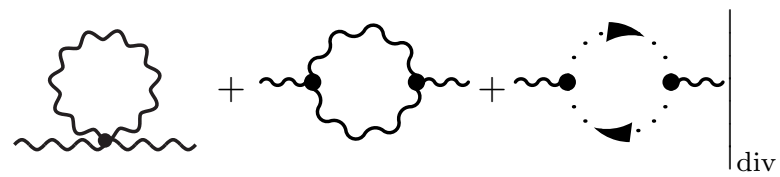

$$
\begin{aligned}
& =\frac{g^{2}}{2} \Xi(p) \operatorname{tr}\left[T^{a} T^{d}\right]\left[-3\left(\frac{1+x}{5} p^{L} p_{L} \eta^{M N}-x p^{M} p^{N}\right)+4\left(p^{L} p_{L} \eta^{M N}-p^{M} p^{N}\right)\right]_{d b} \\
& +3 g^{2} \Lambda^{3} \operatorname{tr}\left[T^{a} T^{d}\right]\left[2\left(\frac{1+x}{5} \eta^{M N}-x \frac{p^{M} p^{N}}{p^{L} p_{L}}\right)-\eta^{M N}\right]_{d b}, \\
& \sim 2 m \\
& =-2 g^{2} \Xi(p) \operatorname{tr}\left[\tau_{\ell}^{a} \tau_{\ell}^{d}\right]\left[-\left(\frac{1+x}{5} p^{L} p_{L} \eta^{M N}-x p^{M} p^{N}\right)+p^{L} p_{L} \eta^{M N}-p^{M} p^{N}\right]_{d b} \\
& -4 g^{2} \Lambda^{3} \operatorname{tr}\left[\tau_{\ell}^{a} \tau_{\ell}^{d}\right]\left[2\left(\frac{1+x}{5} \eta^{M N}-x \frac{p^{M} p^{N}}{p^{L} p_{L}}\right)-\eta^{M N}\right]_{d b},
\end{aligned}
$$

where the external lines have indices of $(M, a)$ and $(N, b)$ and $p_{M}=\left(p_{\mu}, p_{5}+\frac{\theta^{a} T^{a}}{2 \pi R}\right)$ is the external momentum.

The gauge invariance requires

$$
p^{M} p_{M} \Xi(p)(4 x-1)-2(3+8 x) \Lambda^{3}=0 .
$$

Its possible solutions, which are also consistent with eq. (B.12), are

$$
\begin{aligned}
\Lambda^{3} & =0, & x & =\frac{1}{4}, \\
\Xi(p) & =\frac{\Lambda^{3}}{p^{M} p_{M}}, & x & =-\frac{7}{12}, \\
\Xi(p) & =0, & \Lambda^{3} & =0 .
\end{aligned}
$$

The second one does not regularize the integral for $p=0$ and thus is not suitable for regularization. The last one is a special case of the first one.

Thus, we conclude

$$
\Lambda^{3}=0, x=\frac{1}{4}
$$

Notice that, if we use the dimensional regularization, $\Lambda^{3}, x$ and $\Xi(p)$ are explicitly calculated as

$$
\Lambda^{3}=0, x=\frac{1}{4}, \Xi(p)=-\frac{i}{128 \pi} \sqrt{-p^{M} p_{M}} .
$$




\section{Example of the two-loop calculation}

This appendix is dedicated to deduce eq. (4.8), the contribution from the two-loop diagram with a fermion loop;

$$
V_{F, \mathrm{eff}}^{2 L}(\theta)=i \circlearrowleft
$$

First, we apply the Feynman rules to the diagram and get

$$
\begin{aligned}
V_{F, \mathrm{eff}}^{2 L}(\theta)=\frac{i}{2} \frac{1}{2 \pi R} \sum_{n_{1}} \int \frac{d^{4} p}{(2 \pi)^{4}} \frac{1}{2 \pi R} \sum_{n_{2}} \int \frac{d^{4} k}{(2 \pi)^{4}}\left[\frac{-i \eta_{M N}}{p^{2}-\left(\frac{n_{1}}{R}+\frac{\theta^{c} T^{c}}{2 \pi R}\right)^{2}}\right]_{a b} \\
\times(-1) \operatorname{tr}\left[\frac{i}{\not p+\not k-\gamma_{5}\left(\frac{n_{1}+n_{2}}{R}+\frac{\theta^{c} \tau_{\ell}^{c}-\beta_{\ell}}{2 \pi R}\right)} i g \gamma^{M} \tau_{\ell}^{a}\right. \\
\left.\times \frac{i}{\not k-\gamma_{5}\left(\frac{n_{2}}{R}+\frac{\theta^{c} \tau_{\ell}^{c}-\beta_{\ell}}{2 \pi R}\right)} i g \gamma^{N} \tau_{\ell}^{b}\right],
\end{aligned}
$$

where the trace operates on both $\gamma^{M}$ 's and $\tau_{\ell}^{a}$ 's.

Next, we use eq. (4.6) to rearrange the integrand into an analytic function of $\left(\frac{n_{1}}{R}+\frac{\theta^{c} T^{c}}{2 \pi R}\right)$ and $\left(\frac{n_{2}}{R}+\frac{\theta^{c} \tau_{\ell}^{c}-\beta_{\ell}}{2 \pi R}\right)$. After $\tau_{\ell}^{a}$ being shifted to the left, $\operatorname{tr}[\ldots]$ in the above equation becomes

$$
g^{2} \operatorname{tr}\left[\tau_{\ell}^{e}\left(\frac{1}{\not p+\not k-\gamma_{5}\left(\frac{n_{1}+n_{2}}{R}+\frac{\theta^{c} \tau_{\ell}^{c}-\beta_{\ell}+\theta^{c} T^{c}}{2 \pi R}\right)}\right)_{e a} \gamma^{M} \frac{1}{\not k-\gamma_{5}\left(\frac{n_{2}}{R}+\frac{\theta^{c} \tau_{\ell}^{c}-\beta_{\ell}}{2 \pi R}\right)} \gamma^{N} \tau_{\ell}^{b}\right]
$$

where the subscript, ea, shows the indices for $T^{a}$.

Now, we can apply eq. (3.5) to $\left(\frac{n_{1}}{R}+\frac{\theta^{c} T^{c}}{2 \pi R}\right)$ and $\left(\frac{n_{2}}{R}+\frac{\theta^{c} \tau_{\ell}^{c}-\beta_{\ell}}{2 \pi R}\right)$. Notice that we can treat these variables independently since they can be diagonalized simultaneously. We obtain

$$
\begin{array}{r}
V_{F, \mathrm{eff}}^{2 L}(\theta)=\frac{i}{2} \sum_{m_{1}} \int \frac{d^{5} p}{(2 \pi)^{5}} \sum_{m_{2}} \int \frac{d^{5} k}{(2 \pi)^{5}} e^{-i 2 \pi R\left(p_{5} m_{1}+k_{5} m_{2}\right)}\left[e^{i \theta^{c} T^{c} m_{1}}\right]_{a b} \frac{-i \eta_{M N}}{p^{L} p_{L}} \\
\times\left(-g^{2}\right) \operatorname{tr}\left[e^{i\left(\theta^{c} \tau_{\ell}^{c}-\beta_{\ell}\right) m_{2}} \frac{1}{\gamma^{J}(p+k)_{J}} \gamma^{M} \frac{1}{\gamma^{I} k_{I}} \gamma^{N} \tau_{\ell}^{b} \tau_{\ell}^{a}\right] .
\end{array}
$$

Working out the trace of the gamma matrices, we obtain

$$
\begin{aligned}
V_{F, \mathrm{eff}}^{2 L}(\theta)=6 g^{2} & \sum_{m_{1}, m_{2}}\left[e^{i \theta^{c} T^{c} m_{1}}\right]_{a b} \operatorname{tr}\left[e^{i\left(\theta^{c} \tau_{\ell}^{c}-\beta_{\ell}\right) m_{2}} \tau_{\ell}^{b} \tau_{\ell}^{a}\right] \\
& \times \int \frac{d^{5} p}{(2 \pi)^{5}} \int \frac{d^{5} k}{(2 \pi)^{5}} e^{-i 2 \pi R\left(p_{5} m_{1}+k_{5} m_{2}\right)} \frac{(p+k)^{M} k_{M}}{p^{L} p_{L}(p+k)^{J}(p+k)_{J} k^{I} k_{I}} .
\end{aligned}
$$


Open Access. This article is distributed under the terms of the Creative Commons Attribution License (CC-BY 4.0), which permits any use, distribution and reproduction in any medium, provided the original author(s) and source are credited.

\section{References}

[1] ATLAS collaboration, Combined measurements of Higgs boson production and decay using up to $80 \mathrm{fb}^{-1}$ of proton-proton collision data at $\sqrt{s}=13 \mathrm{TeV}$ collected with the ATLAS experiment, ATLAS-CONF-2018-031 (2018) [INSPIRE].

[2] CMS collaboration, Combined measurements of the Higgs boson's couplings at $\sqrt{s}=13 \mathrm{TeV}$, CMS-PAS-HIG-17-031 (2018) [INSPIRE].

[3] M.J.G. Veltman, The Infrared-Ultraviolet Connection, Acta Phys. Polon. B 12 (1981) 437 [INSPIRE].

[4] M. Davier, L. Maiani, C.A. Savoy and J. Scherk, Vector bosons and Higgs bosons in the Weinberg-Salam theory of weak and electromagnetic interactions, proceedings of the Summer School on Particle Physics, Gif-sur-Yvette, France, 3-7 September 1979, National Institute for Nuclear Physics, Paris France (1979) [INSPIRE].

[5] S. Dimopoulos and S. Raby, Supercolor, Nucl. Phys. B 192 (1981) 353 [inSPIRE].

[6] E. Witten, Dynamical Breaking of Supersymmetry, Nucl. Phys. B 188 (1981) 513 [InSPIRE].

[7] M. Dine, W. Fischler and M. Srednicki, Supersymmetric Technicolor, Nucl. Phys. B 189 (1981) 575 [INSPIRE].

[8] D.B. Kaplan and H. Georgi, SU(2) $\times$ U(1) Breaking by Vacuum Misalignment, Phys. Lett. B 136 (1984) 183 [INSPIRE].

[9] D.B. Kaplan, H. Georgi and S. Dimopoulos, Composite Higgs Scalars, Phys. Lett. B 136 (1984) 187 [INSPIRE].

[10] H. Georgi, D.B. Kaplan and P. Galison, Calculation of the Composite Higgs Mass, Phys. Lett. B 143 (1984) 152 [inSPIRE].

[11] T. Banks, Constraints On $\mathrm{SU}(2) \times \mathrm{U}(1)$ Breaking By Vacuum Misalignment, Nucl. Phys. B 243 (1984) 125 [INSPIRE].

[12] H. Georgi and D.B. Kaplan, Composite Higgs and Custodial SU(2), Phys. Lett. B 145 (1984) 216 [INSPIRE].

[13] M.J. Dugan, H. Georgi and D.B. Kaplan, Anatomy of a Composite Higgs Model, Nucl. Phys. B 254 (1985) 299 [INSPIRE].

[14] H. Georgi, A Tool Kit for Builders of Composite Models, Nucl. Phys. B 266 (1986) 274 [INSPIRE].

[15] D.B. Fairlie, Two Consistent Calculations of the Weinberg Angle, J. Phys. G 5 (1979) L55 [INSPIRE].

[16] N.S. Manton, A New Six-Dimensional Approach to the Weinberg-Salam Model, Nucl. Phys. B 158 (1979) 141 [INSPIRE].

[17] P. Forgacs and N.S. Manton, Space-Time Symmetries in Gauge Theories, Commun. Math. Phys. 72 (1980) 15 [inSPIRE]. 
[18] Y. Hosotani, Dynamical Mass Generation by Compact Extra Dimensions, Phys. Lett. B 126 (1983) 309 [INSPIRE].

[19] Y. Hosotani, Dynamical Gauge Symmetry Breaking as the Casimir Effect, Phys. Lett. B 129 (1983) 193 [INSPIRE].

[20] Y. Hosotani, Dynamics of Nonintegrable Phases and Gauge Symmetry Breaking, Annals Phys. 190 (1989) 233 [INSPIRE].

[21] G. von Gersdorff, N. Irges and M. Quirós, Finite mass corrections in orbifold gauge theories, in proceedings of the 37th Rencontres de Moriond on Electroweak Interactions and Unified Theories, Les Arcs, France, 9-16 March 2002, pp. 169-176 [hep-ph/0206029] [InSPIRE].

[22] Y. Hosotani, Dynamical gauge symmetry breaking by Wilson lines in the electroweak theory, in proceedings of the 2004 International Workshop on Dynamical Symmetry Breaking, Nagoya, Japan, 21-22 December 2004, pp. 17-34 [hep-ph/0504272] [INSPIRE].

[23] Y. Hosotani, All-order Finiteness of the Higgs Boson Mass in the Dynamical Gauge-Higgs Unification, hep-ph/0607064 [INSPIRE].

[24] A.T. Davies and A. McLachlan, Congruency Class Effects in the Hosotani Model, Nucl. Phys. B 317 (1989) 237 [InSPIRE].

[25] I. Antoniadis, K. Benakli and M. Quirós, Finite Higgs mass without supersymmetry, New J. Phys. 3 (2001) 20 [hep-th/0108005] [INSPIRE].

[26] N. Maru and T. Yamashita, Two-loop Calculation of Higgs Mass in Gauge-Higgs Unification: 5D Massless QED Compactified on $S^{1}$, Nucl. Phys. B 754 (2006) 127 [hep-ph/0603237] [INSPIRE].

[27] Y. Hosotani, N. Maru, K. Takenaga and T. Yamashita, Two Loop finiteness of Higgs mass and potential in the gauge-Higgs unification, Prog. Theor. Phys. 118 (2007) 1053 [arXiv: 0709.2844] [INSPIRE].

[28] J. Heffner and H. Reinhardt, Finite-temperature Yang-Mills theory in the Hamiltonian approach in Coulomb gauge from a compactified spatial dimension, Phys. Rev. D 91 (2015) 085022 [arXiv: 1501.05858] [INSPIRE].

[29] H. Reinhardt, Hamiltonian finite-temperature quantum field theory from its vacuum on partially compactified space, Phys. Rev. D 94 (2016) 045016 [arXiv:1604.06273] [inSPIRE].

[30] M.M. Anber and T. Sulejmanpasic, The renormalon diagram in gauge theories on $\mathbb{R}^{3} \times \mathbb{S}^{1}$, JHEP 01 (2015) 139 [arXiv:1410.0121] [INSPIRE].

[31] K. Ishikawa, O. Morikawa, A. Nakayama, K. Shibata, H. Suzuki and H. Takaura, Infrared renormalon in the supersymmetric $\mathbb{C} P^{N-1}$ model on $\mathbb{R} \times S^{1}$, arXiv:1908.00373 [INSPIRE].

[32] L.F. Abbott, The Background Field Method Beyond One Loop, Nucl. Phys. B 185 (1981) 189 [INSPIRE]. 